\title{
EDITORIAL
}

\section{Are you pulling my airway?}

\author{
C.Y. Seow
}

들 xcessive airway narrowing is a hallmark of asthma. This is due to unencumbered shortening of airway smooth muscle, leading to severe airways obstruction and even closure of airways. Remarkably, in healthy subjects, this does not occur, and there is little doubt that the explanation of this apparent "protection" in normal subjects will be essential for our understanding of asthma. Parenchymal attachment to the adventitial wall of the airways in the lung is widely believed to contribute, at least in part, to the loads seen by the airway smooth muscle, and, hence, it is assumed to be a significant factor in limiting airway narrowing in healthy individuals [113]. In this issue of the European Respiratory Journal, NoBLE et al. [14] have raised questions on this common belief. In a carefully designed experiment, they found, in mid-sized porcine bronchi, that the force pulling on the smooth muscle layer by the parenchyma surrounding the adventitial airway wall was insignificant and that it did not restrict lumen narrowing of the airway. The results suggest that parenchymal tethering may not play an important role in the altered airway calibre and smooth muscle function observed at different lung volumes and some disease states.

Comparing airway narrowing in its intact in vivo environment and airway narrowing in an isolated bronchial segment is not a small feat. First, one has to make sure that the same generation of bronchi are compared. Secondly, the geometric distortion of airways at different lung volumes has to be mimicked in vitro. Thirdly, the history of stress and strain experienced by the airway smooth muscle in vivo, up to the point of testing, has to be replicated in the in vitro measurement. Finally, the degree of muscle activation and transmural pressure in the bronchial segments must be comparable to those in vivo, in order to ensure a valid comparison. Taking these provisions into account, NOBLE et al. [14] set out to measure the lumen diameter of bronchi of in vivo and in vitro groups as functions of transpulmonary and transmural pressure, respectively; for both groups, identical results were obtained under both resting and activated conditions.

To many investigators in the field, this finding is surprising, at least at a first glance. One implication of this finding is that uncoupling of parenchyma from airways may not significantly change the elastic load on airway smooth muscle, and, therefore, a loss or attenuation of parenchymal interdependence should not be considered a good candidate contributor to airway hyperresponsiveness. Another implication is that lung

CORRESPONDENCE: C.Y. Seow, Dept of Pathology and Laboratory Medicine, The James Hogg iCAPTURE Centre for Cardiovascular and Pulmonary Research, St. Paul's Hospital, University of British Columbia, 166-1081 Burrard Street, Vancouver, BC V6Z 1Y6, Canada. Fax: 16048069274 E-mail: cseow@mrl.ubc.ca volume change may not directly lead to changes in airway smooth muscle length, with a corollary that the changes in lung function associated with a change in lung volume may not be interpreted in terms of stretch-induced alterations in airway smooth muscle contractility. Changes in lung volume associated with tidal breathing and deep inspiration are known to affect airway calibre and airway smooth muscle function $[4,7,13,15-27]$. In interpreting these results, it is often assumed, explicitly or implicitly, that the effect is mediated through lung parenchyma that tethers the airway wall to the rest of the lung. In light of the findings by NobLE et al. [14], should these results be reinterpreted?

Before the implications of the findings of NOBLE et al. [14] are overextended, the limitations of their study should be examined. The choice of porcine airways in this particular study may not be the best, if the findings are to be relevant to human lung physiology. At 10th generation, porcine bronchi probably contain more cartilage than human bronchi at the same generation [28]. The stiffer porcine airways may have prevented parenchymal tethers from altering the adventitial diameter of the airways; therefore, the decrease in lumen diameter in constricted airways represents decoupling of the smooth muscle layer from the adventitia, as speculated by NOBLE et al. [14]. The choice of animal species may underlie the discrepancies between the results reported by NOBLE et al. [14] and those reported by others [1-13, 15-27] that suggest a significant role for parenchymal tethering in maintaining airway patency. The outer diameter of the airway segment during constriction was not measured by NOBLE et al. [14]. Such a measurement would allow us to discern two possible interpretations of their results, one being that the adventitial wall is too stiff to deform, even in the presence of substantial lumen narrowing, and the other being that the parenchymal tethering is too compliant to provide any significant load on the constricting muscle layer. In either case, their results suggest that in mid-sized airways of pig lung, airway smooth muscle is insulated from mechanical perturbations mediated through the parenchyma.

One obvious remedy for the limitations associated with the studies of NOBLE et al. [14] is to repeat the experiments in a species that has noncartilaginous mid-sized airways. Would a more compliant noncartilaginous airway be more amenable to parenchymal pulling, to the extent that the elastic load can be transmitted to the smooth muscle layer without much hindrance? There is strong evidence that deep inspiration in humans is associated with dilatation of partially constricted airways [29]. Such dilatation is less certain in small airways. How important, then, is the airway-parenchymal interdependence in maintaining patency in small airways? A related question is how surfactant release after a big breath [30] 
affects small airway impedance. Maybe airway impedance is little affected by the direct stretching of airway smooth muscle by parenchyma, but, instead, through the release of mediators like nitric oxide [31]. Answers to these questions will help us to more accurately interpret data from in vivo and in vitro experiments that examine the importance of parenchymal tethering and lung volume change on airway responsiveness.

A major contribution from NOBLE et al. [14] is the establishment of a creative protocol for comparing airway narrowing with and without parenchyma. The numerous control measurements as part of their protocol are essential for a meaningful comparison. The protocol set by NOBLE et al. [14] should be followed in future studies of a similar type. However, one may extend it to include additional measurements. For example, the viscoelastic properties of parenchyma (in the form of shear modulus [18]), instead of just elastic properties [14], should provide valuable insights into the (more realistic) role of parenchyma in the dynamic environment of the lung. The study by NOBLE et al. [14] only deals with static properties, and it should be kept in mind that parenchyma exerts its effect in a mechanically dynamic environment. Under static conditions, the elastic component of parenchyma may be too compliant to effectively stretch the smooth muscle layer in an airway. Under dynamic conditions, the viscous component of parenchyma should facilitate force transmission to the muscle layer, especially when the rate of lung volume change is high. Therefore, even if the pure elastic load provided by the parenchyma is too small to make a difference in lumen diameter under static conditions, the viscoelastic properties of the parenchyma under dynamic conditions may allow transient loads to be transmitted to the muscle layer.

The topic of airway-parenchymal interdependence is of great interest to many of us who are interested in identifying factors that influence airway patency in health and disease. It should be pointed out that NOBLE et al. [14] are not the first to question the significance of the interdependence $[32,33]$. Perhaps it is time to take a closer look at this important issue, and to reassess the significance of parenchymal distortion as a source of mechanical force limiting airway narrowing and the likelihood of a weakened tethering force as a contributor in the pathogenesis of asthma. If we don't understand the normal airway, how can we in asthma?

\section{REFERENCES}

1 Sasaki H, Takishima T, Sasaki T. Influence of lung parenchyma on dynamic bronchial collapsibility of excised dog lungs. J Appl Physiol 1977; 42: 699-705.

2 Suzuki S, Sasaki H, Sekizawa K, Takishima T. Isovolume pressure-flow relationships in intrapulmonary bronchi of excised dog lungs. J Appl Physiol 1982; 52: 295-303.

3 Ding DJ, Martin JG, Macklem PT. Effects of lung volume on maximal methacholine-induced bronchoconstriction in normal humans. J Appl Physiol 1987; 62: 1324-1330.

4 Gunst SJ, Warner DO, Wilson TA, Hyatt RE. Parenchymal interdependence and airway response to methacholine in excised dog lobes. J Appl Physiol 1988; 65: 2490-2497.

5 Macklem PT. Mechanical factors determining maximum bronchoconstriction. Eur Respir J 1989; 2: Suppl. 6, 516s-519s.
6 Okazawa M, Bai TR, Wiggs BR, Pare PD. Airway smooth muscle shortening in excised canine lung lobes. J Appl Physiol 1993; 74: 1613-1621.

7 Moreno RH, Lisboa C, Hogg JC, Pare PD. Limitation of airway smooth muscle shortening by cartilage stiffness and lung elastic recoil in rabbits. J Appl Physiol 1993; 75: 738-744.

8 Adler A, Cowley EA, Bates JH, Eidelman DH. Airwayparenchymal interdependence after airway contraction in rat lung explants. J Appl Physiol 1998; 85: 231-237.

9 Lopez-Aguilar J, Romero PV. Effect of elastase pretreatment on rat lung strip induced constriction. Respir Physiol 1998; 113: 239-246.

10 Okazawa M, D'Yachkova Y, Pare PD. Mechanical properties of lung parenchyma during bronchoconstriction. J Appl Physiol 1999; 86: 496-502.

11 Salerno FG, Ludwig MS. Elastic moduli of excised constricted rat lungs. J Appl Physiol 1999; 86: 66-70.

12 Okazawa M, Pare PD, Lambert RK. Compliance of peripheral airways deduced from morphometry. J Appl Physiol 2000; 89: 2373-2381.

13 Irvin CG, Pak J, Martin RJ. Airway-parenchyma uncoupling in nocturnal asthma. Am J Respir Crit Care Med 2000; 161: 50-56.

14 Noble PB, Sharma A, McFawn PK, Mitchell HW. Airway narrowing in porcine bronchi with and without lung parenchyma. Eur Respir J 2005; 26: 804-811.

15 Dandurand RJ, Xu LJ, Martin JG, Eidelman DH. Airwayparenchymal interdependence and bronchial responsiveness in two highly inbred rat strains. J Appl Physiol 1993; 74: 538-544.

16 Nagase T, Martin JG, Ludwig MS. Comparative study of mechanical interdependence: effect of lung volume on Raw during induced constriction. J Appl Physiol 1993; 75: 2500-2505.

17 Brown RH, Mitzner W. Effect of lung inflation and airway muscle tone on airway diameter in vivo. J Appl Physiol 1996; 80: 1581-1588.

18 Lambert RK, Pare PD. Lung parenchymal shear modulus, airway wall remodeling, and bronchial hyperresponsiveness. J Appl Physiol 1997; 83: 140-147.

19 Shen X, Gunst SJ, Tepper RS. Effect of tidal volume and frequency on airway responsiveness in mechanically ventilated rabbits. J Appl Physiol 1997; 83: 1202-1208.

20 Tepper RS, Wiggs B, Gunst SJ, Pare PD. Comparison of the shear modulus of mature and immature rabbit lungs. $J$ Appl Physiol 1999; 87: 711-714.

21 Okazawa M, D’Yachkova Y, Pare PD. Mechanical properties of lung parenchyma during bronchoconstriction. J Appl Physiol 1999; 86: 496-502.

22 Salerno FG, Shinozuka N, Fredberg JJ, Ludwig MS. Tidal volume amplitude affects the degree of induced bronchoconstriction in dogs. J Appl Physiol 1999; 87: 1674-1677.

23 Adler A, Bates JH. A micromechanical model of airwayparenchymal interdependence. Ann Biomed Eng 2000; 28: 309-317.

24 Brown R, Mitzner W. Effects of tidal volume stretch on airway constriction in vivo. J Appl Physiol 2001; 91: 1995-1998.

25 Gunst SJ, Shen X, Ramchandani R, Tepper RS. Bronchoprotective and bronchodilatory effects of deep 
inspiration in rabbits subjected to bronchial challenge. I Appl Physiol 2001; 91: 2511-2516.

26 Brown RH, Mitzner W. Airway response to deep inspiration: role of inflation pressure. J Appl Physiol 2001; 91: 2574-2578.

27 Brown RH, Mitzner W. Duration of deep inspiration and subsequent airway constriction in vivo. J Asthma 2003; 40: 119-124.

28 Mukherjee A, Wasserman MA. Role of alpha-adrenergic receptors in cartilaginous and non-cartilaginous human airways. Methods Find Exp Clin Pharmacol 1986; 8: 667-673.

29 Brown RH, Scichilone N, Mudge B, Diemer FB, Permutt S, Togias A. High-resolution computed tomographic evaluation of airway distensibility and the effects of lung inflation on airway caliber in healthy subjects and individuals with asthma. Am J Respir Crit Care Med 2001; 163: 994-1001.

30 Dietl P, Frick M, Mair N, Bertocchi C, Haller T. Pulmonary consequences of a deep breath revisited. Biol Neonate 2004; 85: 299-304.

31 Brown RH, Mitzner W. Airway response to deep inspiration: role of nitric oxide. Eur Respir J 2003; 22: 57-61.

32 Olson LE. Small airway pressure-diameter relationships during nonhomogeneous lung lobe inflation. J Appl Physiol 1987; 62: 2377-2382.

33 Olson LE. Regional compliance and bronchial pressurediameter relationships in excised pig lungs. Respir Physiol 1991; 86: 25-39. 